Caligrama, Belo Horizonte, v. 22, n. 2, p. 33-60, 2017

\title{
Analisi di una conversazione tratta da un dominio professionale: la gestione di una discussione fra parlanti d'italiano
}

\section{Analysis of a conversation from a professional domain: the management of a discussion among speakers of Italian}

Roberta Ferroni

Universidade de São Paulo, São Paulo, São Paulo / Brasil robertaferronibr@gmail.com

Riassunto: a partire da un dialogo tratto da un corpus composto da conversazioni faccia a faccia, raccolte all'interno di un dominio professionale, tra parlanti italiani, l'articolo esplora le potenzialità che gli studi conversazionali possono offrire per identificare le strategie discorsive, usate nel corso di una discussione. L'analisi ha permesso di mettere a fuoco una vasta gamma di meccanismi conversazionali come: discorsi simultanei, sovrapposizioni, interruzioni e segnali discorsivi metatestuali e modulatori, che tessono la trama dell'interazione e puntellano gli interventi dei parlanti, al fine di affermare e controbattere, in maniera più o meno accesa, le proprie opinioni. Si conclude che l'input proveniente dalla vita reale, associato a percorsi di osservazione, costruiti ad hoc, in base a quelli che sono i meccanismi osservati nell'ambito degli studi della conversazione, possono effettivamente costituire delle valide risorse, da usare in classe, per sensibilizzare gli apprendenti allo sviluppo della competenza discorsiva in italiano LS.

Parole-chiave: conversazione faccia a faccia; input autentico; competenza discorsiva. 
Abstract: From a dialogue taken from a corpus composed of face-toface conversations between Italian speakers, collected in a professional environment, the article explores the potential that conversational studies can provide to identify the discursive strategies used during a discussion. The analysis allowed to focus on a wide range of conversational mechanisms such as simultaneous speeches, overlaps, interruptions and discursive meta-textual signals and modulators, that weave the threads of interaction and support the interventions of the speakers in order to affirm and refute, more or less lively, their opinions. It concludes that the input from the real life, associated with observations built ad hoc, based on the mechanisms observed in the context of the studies of conversation, may indeed be valuable resources for use in the classroom, to sensitize learners to the development of discursive competence in Italian as a foreign language.

Keywords: face-to-face conversations; authentic input; discursive competence.

Recebido em: 10 de março de 2017.

Aprovado em: 11 de maio de 2017.

\section{Introduzione}

In una conferenza tenutasi in occasione del III convegno ILSA ${ }^{1}$ nel lontano 1996, Daniela Zorzi, nota studiosa di Analisi della Conversazione (d'ora in poi AC) applicata alla didattica dell'italiano lingua seconda (d'ora in poi L2), prendeva atto del fatto che molti dei materiali didattici specializzati nell' insegnamento dell'italiano lingua seconda (d'ora in poi L2) e lingua straniera (d'ora in poi LS), specie di livello avanzato, erano poco sensibili ai tratti conversazionali che contraddistinguono l'oralità come, ad esempio, auto ed eterointerruzioni, sovrapposizioni, auto ed eterocorrezioni e ancora cambi di topico, ripetizioni e segnali discorsivi. Zorzi si auspicava che, in un futuro non troppo lontano, questi fenomeni potessero trovare una giusta collocazione nella didattica dell'italiano L2 e LS, visto che "è solo conoscendo le caratteristiche della comunicazione

\footnotetext{
${ }^{1}$ Insegnanti Italiano Lingua Seconda Associati.
} 
reale che si potrà aiutare lo studente ad usare la lingua in modo adeguato ai diversi contesti" (ZORZI, 1996, p. 15). A distanza di circa vent'anni la situazione, come salientano Gillani e Pernas (2014), è rimasta pressoché immutata $\mathrm{e}$-salvo sporadiche proposte operative che mirano a sviluppare una pedagogia propensa alla "co-costruzione della conversazione, all'alternanza e all'interruzione" (PERNAS et al., 2011, p.66) ${ }^{2}$ - molti dei manuali d'italiano L2 e LS di livello avanzato continuano a dimostrarsi ignari dei meccanismi prototipici che regolano il parlato.

Fatta questa premessa l'articolo ha lo scopo di illustrare il progetto di ricerca intitolato "L'acquisizione dei segnali discorsivi nel parlato dialogico di apprendenti di italiano LS - di livello intermedio e avanzato - attraverso l'approccio conversazionale"3 e il cui obiettivo è consolidare, fra apprendenti d'italiano LS, consapevolezza e capacità d'uso relativamente all'utilizzo dei segnali discorsivi. In particolare, in queste pagine ci soffermeremo a descrivere la $1^{a}$ fase del progetto, che consiste appunto nella creazione di una piattaforma on line denominata Ma dai!: proposte didattiche in azione per l'apprendimento dei segnali discorsivi in italiano $L S$, composta da conversazioni faccia a faccia, raccolte in situazioni sia simmetriche che asimmetriche, tra parlanti italiani. In una $2^{a}$ fase le interazioni serviranno come input per elaborare delle attività didattiche, da usare in classe, che consentano a studenti di italiano LS di livello avanzato di apprendere i meccanismi e le procedure tipiche della conversazione.

Dopo aver illustrato i criteri impiegati per l'elaborazione del corpus che comporrà la piattaforma, effettueremo, tramite gli studi conversazionali (SACKS; SCHEGLOFF; JEFFERSON, 1974; BAZZANELLA, 1994, 1995, 2011), l'analisi di una microscena, tratta dallo stesso corpus, al fine di mettere a fuoco le strategie discorsive, usate da parlanti di italiano, nel corso di una discussione.

Scopo del seguente contributo sarà vedere quali informazioni si possono ricavare, che siano poi applicabili in una fase successiva del

\footnotetext{
${ }^{2}$ Si vedano la sequenza didattica illustrata da Pugliese (2015) che presenta delle attività per favorire lo sviluppo e la capacità d'uso del segnale discorsivo figurati e 1'unità 6 del corso d'italiano per stranieri Bravissimo! 3-B1 (Casa delle Lingue), ricca di spunti per l'insegnamento della dimensione testuale dello scambio conversazionale (FERRONI; BIRELLO, 2016).

${ }^{3}$ Il progetto è finanziato dall'ente brasiliano FAPESP (2016/08917-9).
} 
progetto, ${ }^{4}$ dallo studio di un'interazione simmetrica raccolta all'interno di un dominio professionale, attraverso l'apparato metodologico proposto dagli studi conversazionali.

\section{Genesi della piattaforma Ma dai!: i criteri e la raccolta dati}

Negli ultimi anni, gli insegnanti più avveduti, per sensibilizzare i propri studenti a quelle che sono le regole che gestiscono la cocostruzione conversazionale in italiano L2 e LS, possono contare su una serie di materiali d'appoggio e di facile reperibilità, come, ad esempio, sequenze tratte da film, programmi tv, fino a delle verie e proprie banche dati che compongono i cosiddetti corpora di lingua orale. ${ }^{5}$ L'uso di questi materiali non riesce tuttavia a soddisfare pienamente quelli che sono i bisogni del pubblico che si ha di fronte. Si pensi, ad esempio, ai film o a programmi televisivi, che pur essendo materiali non pensati per scopi didattici, spesso e volentieri, riproducono delle conversazioni che rispecchiano solo parzialmente le modalità della lingua parlata, dato che gli "attori" recitano sulla base di un copione scritto che può essere interpretato più o meno liberamente. Anche i corpora di lingua parlata presentano, a loro volta, una serie di problemi. Infatti, pur restituendo degli stralci di conversazioni tratte dalla vita reale, possono proporre situazioni quotidiane molto lontane da quelle che i nostri allievi potrebbero dover gestire una volta fuori dall'aula. Nel caso poi si tratti di corpora supportati solo da dati audio, sono da valutare probabili errori di fraintendimento che l'assenza di immagini può provocare, specie agli occhi di un parlante straniero, poiché anche il linguaggio del corpo, alla stregua di quello verbale, contribuisce a tutti gli effetti i fini dell'andamento dell'evento comunicativo (CONTENTO, 1999; BONAIUTO; MARICCHIOLO, 2003; POGGI, 2006; CILIBERTI, 2015). ${ }^{6}$ Nel tentativo di sciogliere questi interrogativi che, come docente di italiano LS, accompagnano la nostra prassi didattica, a partire da marzo del 2017 è in fase di sviluppo il progetto denominato "L'acquisizione dei segnali discorsivi nel parlato

\footnotetext{
${ }^{4}$ Come già anticipato la finalità del progetto consiste nello sviluppare la competenza discorsiva fra apprendenti di italiano LS di livello intermedio e avanzato.

${ }^{5}$ Per le lingue romanze si veda il corpus C-ORAL-ROM.

${ }^{6}$ Sui vantaggi relativi all'uso di materiali multimodali si vedano, fra gli altri, Moreno Jaén; Pérez Basanta (2009).
} 
dialogico di apprendenti di italiano LS - di livello intermedio e avanzato - attraverso l'approccio conversazionale". Come anticipato nel paragrafo precedente il progetto, ancora in fase embrionale, prevede, nel corso della tappa iniziale, la creazione di una piattaforma multimodale, corredata da dati audio e video, che consenta all'utente di navigare all'interno di "microscene" che riproducono interazioni faccia a faccia, raccolte in situazioni sia simmetriche che asimmetriche, fra parlanti di italiano e accompagnate dalla rispettiva trascrizione. ${ }^{7}$

Ai fini della messa a punto di questa prima fase che servirà come input per alimentare la creazione di materiali specifici per lo sviluppo della competenza interazionale ${ }^{8}$ in italiano LS fra apprendenti di livello avanzato, è stato necessario fissare dei criteri che qui di seguito passeremo ad illustrare.

Il materiale video che comporrà la piattaforma è stato raccolto 9 dalla ricercatrice ed è composto da un campione di "microscene", corrispondenti a 10 ore di conversazioni faccia a faccia. ${ }^{10} \mathrm{Con}$ la speranza di poter venire incontro alle esigenze dei destinatari di questo progetto, abbiamo preferito controllare una serie di variabili. ${ }^{11}$ Dal punto di vista

${ }^{7}$ La piattaforma sarà poi disponibile online.

${ }^{8}$ Alcuni autori parlano di competenza interazionale (si vedano, per esempio, YOUNG, 2011; PEKAREK DOEHLER; POCHON-BERGER, 2015), altri di competenza discorsiva (QCER, 2002). In questo articolo useremo indistintamente i due termini per riferirci a "a knowledge of rhetorical scripts, a knowledge of certain lexis and syntactic patterns specific to the practice, a knowledge of how turns are managed, a knowledge of topical organization, and a knowledge of the means for signaling boundaires between practices and transitions within the practice itself" (YOUNG, 2011 p. 429).

${ }^{9} \mathrm{La}$ fase di raccolta è avvenuta, attraverso l'ausilio di una telecamera, durante il mese di luglio del 2016.

${ }^{10}$ Per conversazione non intendiamo "indicare quella particolare pratica che è il conversare per diletto, in cui il parlare informalmente tra pari è il mezzo e il fine dell'interazione" bensì ci riferiamo "a un'intera classe di fenomeni che coinvolgono genericamente l'attività di parola" (GALATOLO; PALLOTTI, 1999, p. 14-15).

${ }^{11}$ Chi vi scrive è docente di lingua italiana presso il corso di Laurea di Lingua e Letteratura Italiana della Facoltà di Filosofia, Lettere e Scienze Umane dell'Università di San Paolo del Brasile. Il corso è generalmente frequentato da un pubblico abbastanza eterogeneo sia per età che per interessi. Si tratta di studenti brasiliani, con un'età compresa fra un minimo di 20 e un massimo di 50 anni, e che, oltre allo studio, possono svolgere attività lavorative. 
spaziale, trattandosi di registrazioni effettuate "dal vero", per ridurre il più possibile l'impatto provocato dai rumori ambientali, che inevitabilmente circondano il mondo esterno, abbiamo privilegiato solo le interazioni che si svolgono all'interno di ambienti chiusi. ${ }^{12}$ Questa scelta ci ha indotto a escludere, almeno per il momento, tutta una serie di spazi pubblici (come, ad esempio, strade, negozi e aule). ${ }^{13}$ Sempre per lo stesso motivo il numero delle persone coinvolte nello scambio interattivo è composto da un minimo di 2 a un massimo di 4 membri che partecipano più $o$ meno attivamente alla conversazione. In questo modo abbiamo potuto controllare possibili sovrapposizioni di voci. Questa variabile è stata a sua volta determinante per la sceta dei domini; infatti abbiamo scartato il dominio pubblico, che è quello in cui " 1 'individuo agisce come membro della società" (Quadro Comune Europeo di Riferimento per le Lingue - QCER -, 2002, p. 58), e ci siamo invece concentrati su: dominio personale, quello, cioè, in cui "l'individuo vive come soggetto privato, ed è centrato sulla vita domestica con la famiglia con gli amici" (QCER, 2002, p. 58); dominio professionale "in cui l'individuo è impegnato nel lavoro o nella professione" (QCER, 2002, p. 58); dominio educativo dove "l'individuo è impegnato in attività di apprendimento" (QCER, 2002, p. 58). Nella scelta dei domini ha giocato un ruolo fondamentale anche il profilo dei nostri apprendenti che, lo ricordiamo, sono i principali destinatari di questo progetto. Tenendo conto anche dei descrittori proposti dal QCER (2002), ${ }^{14}$ abbiamo cercato di proporre, oltre ad una serie di situazioni comunicative verosimili a quelle che i nostri studenti potrebbero affrontare una volta fuori dall'aula in lingua italiana, delle situazioni che presuppongono, in termini di partecipazione personale, un certo coinvolgimento. Dal punto di vista diastratico, abbiamo preso come campione delle iterazioni che coinvolgono parlanti, di ambo i sessi, in possesso del diploma di istruzione secondaria di $2^{\circ}$ grado e del diploma di laurea, con età compresa fra i 20 e i 50 anni. Considerando la complessa

\footnotetext{
${ }^{12}$ Per ambienti al chiuso intendiamo abitazioni private e uffici.

${ }^{13}$ Oltre ai rumori di sottofondo, le registrazioni realizzate in ambienti pubblici, presentano una serie di altri inconvenienti. Pensiamo, ad esempio, alla difficoltà di poter disporre delle richieste di autorizzazione.

${ }^{14}$ Secondo i descrittori proposti dal QCER (2002) i parlanti, compresi fra i livelli B2 e C2, devono essere in grado di prendere parte a conversazioni su argomenti familiari, di interesse personale o riguardante la vita quotiodiana e di formulare idee e opinioni con precisione e collegate a quelle degli altri.
} 
situazione geolinguistica italiana, per evitare possibili interferenze con varietà dialettali, le riprese sono state effettuate fra parlanti toscani.

Passiamo ora a descrivere, in modo più approfondito, il dominio professionale prescelto. ${ }^{15}$

Come contesto favorevole alla realizzazione delle riprese è stato individuato il backstage del festival di teatro contemporaneo intitolato "Kilowatt, l'energia del nuovo teatro". ${ }^{16}$ Il festival dedicato alle compagnie emergenti della scena contemporanea (teatro, danza, arti performative, musica, letteratura, arti visive) avviene ogni anno, durante il mese di luglio, presso la città di Sansepolcro, ${ }^{17}$ ed è preceduto da un periodo in cui gli operatori che fanno parte dello staff organizzativo, ${ }^{18}$ svolgono, presso la sede principale del festival, attività lavorative di vario carattere, finalizzate all'organizzazione dell'evento.

Questo contesto ci è parso particolarmente significativo in quanto gli operatori che vi collaborano hanno un profilo molto simile a quello dei nostri studenti di italiano LS: hanno un'età compresa fra i 20 e 40, possiedono un livello di istruzione superiore, inoltre sono impegnati a svolgere una serie di mansioni piuttosto diversificate che vanno dalla gestione della contabilità, all'aggiornamento dei social midia, a riunioni di lavoro con tecnici, fotografi e addetti al settore ospitalità, fino a pranzi informali di lavoro.

Prima di iniziare le riprese, la ricercatrice era entrata in contatto, di persona, con il direttore artistico del festival che, dopo essere stato informato sul motivo della ricerca, ha autorizzato la raccolta dei dati, oltre a fornire una serie di importanti dettagli sul tipo di attività svolte nei giorni precedenti al festival.

\footnotetext{
${ }^{15}$ I dati provenienti dal dominio personale ed educativo sono ancora in fase di raccolta. ${ }^{16}$ Iniziativa che ha ottenuto vari e prestigiosi premi, fra i quali, il Premio Ubu e il Premio Nico Garrone. Per ulteriori approfondimenti su questo evento si rimanda al sito: $<$ http://www.kilowattfestival.it/ >.

${ }^{17}$ Città localizzata in provincia di Arezzo (Toscana) e confinante con Umbria, Romagna e Marche.

${ }^{18}$ Fanno parte dello staff organizzativo: il direttore artistico, la direttrice amministrativa e contabile, il direttore organizzativo e fundraising, il direttore tecnico, il responsabile della segreteria organizzativa e biglietteria, il responsabile promozione, volontari e social-media, l'addetta all'ufficio stampa, la segreteria organizzativa, la segreteria amministrativa, la squadra tecnica, i fotografi del festival, i videomakers. Colgo l'occasione per ringraziare tutti i partecipanti alla ricerca per la disponibilità dimostrata.
} 
Durante il primo giorno delle riprese, il direttore ha presentato la ricercatrice al resto dei membri, informandoli sul motivo della ricerca. Immediatamente dopo le riprese, i partecipanti sono stati invitati a compilare dei questionari, grazie ai quali si è potuto tracciare un quadro socio-linguistico di ognuno. ${ }^{19}$

I dati sono stati raccolti con l'ausilio di una videocamera, poggiata su un treppiede e posizionata in un angolo fisso della stanza in cui si svolgeva l'interazione. I soggetti sono stati informati previamente sulla finalità della ricerca e hanno firmato una liberatoria per consentire la circolazione dei dati e delle immagini.

La maggior parte di quelle che abbiamo definito "microscene" ha una durata non superiore ai tre minuti. Ogni "microscena" si sviluppa intorno ad un preciso compito comunicativo. Per compito intendiamo "un'azione finalizzata che l'individuo considera necessaria per raggiungere un determinato risultato nell'ambito di un problema da risolvere, un impegno da adempiere o un obiettivo da raggiungere" (QCER, 2002, p. 12). Nel caso specifico, abbiamo estrapolato varie interazioni simmetriche che ruotano attorno a precisi compiti comunicativi, come, ad esempio: lavoro di gruppo per organizzare un evento, gestione dei rendiconti amministrativi, gestione del settore ospitalità, gestione e organizzazione del settore social-media.

Per quanto riguarda la modalità di osservazione che abbiamo adottato si pone a metà fra i due poli che Duranti (1997, p. 99) definisce come "partecipazione passiva" e "partecipazione completa". Infatti abbiamo preso parte alle interazioni solo quando i partecipanti ci hanno invitati a intervenire. A questo proposito è importante precisare che pur essendo state registrate fra interlocutori consapevoli, le interazioni non mancano certo di spontaneità, dato che la presenza dell'osservatore non crea un contesto falso, ma semplicemente crea un nuovo contesto che dovrà essere studiato come tale.

Successivamente le microscene sono state trascritte. Come norme per la trascrizione sono state adottate quelle suggerite da Saks, Schegloff

\footnotetext{
${ }^{19}$ Per motivi di spazio ci limiteremo a descrivere il profilo di tre dei collaboratori.
} 
e Jefferson (1974) $)^{20}$ che ci consentono di mettere in risalto una serie di fenomeni conversazionali, oggetto di studio della presente ricerca.

\section{Contesto e metodologia}

Prima di descrivere la metodologia, per contestualizzare l'evento comunicativo, è necessario tracciare un breve profilo di ciascun partecipante e descrivere il contesto in cui ha avuto luogo l'interazione.

L'episodio vede come protagonisti principali Massimo, Gianluca e, nella parte finale, anche un intervento di Luca. ${ }^{21}$

Massimo e Luca hanno 42 anni, mentre Gianluca 35. Sono tutti italiani e possiedono un diploma di laurea. Massimo è direttore

${ }^{20}$ Simboli utilizzati:

, pausa breve;

(.) pausa media;

(1.5) pausa lunga, misurata in secondi e decimi di secondo;

= due segmenti di parlato che si susseguono senza interruzione;

[ punti in cui inizia una sovrapposizione tra i turni di due parlanti;

(parola) segmento di interpretazione incerta;

(all the/other) interpretazioni alternative di un segmento non ben comprensibile;

( ) segmento incomprensibile;

(2 sill) segmento incomprensibile (con indicazione del numero approssimativo di sillabe);

(( )) fra doppie parentesi sono inseriti elementi non verbali (es. gesti, espressioni del viso, postura, sguardo) o informazioni su ciò che avviene durante la conversazione (es. spostamenti, manipolazione di oggetti, avvenimenti di vario genere);

so:: allungamento della sillaba;

? intonazione ascendente (non necessariamente interrogativa);

$\mathrm{hm} / \mathrm{mh}$ vocalizzi indicanti esitazione, assenso, dubbio, ecc.;

$\mathrm{mhm} /$ ehe / aha risate;

capi- parola o frase interrotta bruscamente;

testo pronuncia enfatica;

TESTO volume più alto rispetto al resto del parlato;

$<$ parola $>$ segmento pronunciato a un ritmo più lento;

${ }^{\circ}$ parola ${ }^{\circ}$ volume più basso rispetto al resto del parlato;

testo fenomeno che si desidera mettere in evidenza.

${ }^{21}$ Per motivi di privacy i nomi dei soggetti sono stati modificati. 
organizzativo e fundraising del festival, Gianluca cura la promozione e i social-media, Luca dirige ed è ideatore del festival.

La scena, della durata di 3 minuti, si svolge nella sala adiacente al teatro. La stanza ha un soffitto molto grande, per questo motivo la qualità dell'acustica non è perfetta, pertanto ci sono delle parti che in fase di trascrizione sono, purtroppo, risultate incomprensibili. Ai lati della stanza principale, che nei giorni che precedono il festival funge da area di lavoro, sono disposte due grandi tavole. In una si trova seduto Massimo e nell'altra Gianluca e Luca. Trattandosi un lavoro di équipe è molto frequente che i colleghi si trovino a discutere fra loro per risolvere vari aspetti logistici legati al festival, come accade in questo esempio. Massimo è stato convocato al tavolo di Gianluca per stabilire il numero delle sedie destinato ad ogni spettacolo.

Nel corso di tutta la scena Massimo si trova in piedi, disposto frontalmente rispetto a Gianluca che è seduto dietro al tavolo con davanti il computer. Accanto a Gianluca si trova Luca che sta lavorando silenziosamente al computer. Si veda l'immagine 1 .

Immagine 1

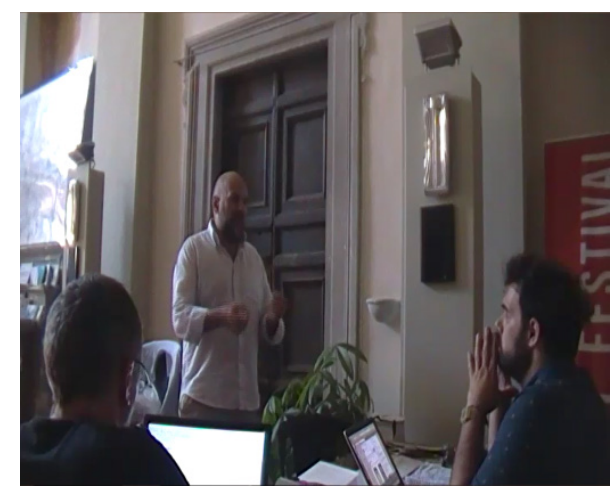

Gianluca, rispetto a Massimo, che lavora da poco più di un anno per Kilowatt, ha già organizzato varie edizoni del festival, per cui possiede molta più esperienza che ostenterà, attraverso una serie di strategie discorsive, nel corso dell'interazione.

Abbiamo scelto di analizzare questo esempio perché, come vedremo, a causa del contrasto di opinioni, la trama discorsiva risulta essere molto ricca sul piano conversazionale. 
L'interazione è composta da ben 44 turni, abbastanza corposi, così distribuiti: 19 turni Gianluca, 17 Massimo e 8 Luca. La lunghezza è attribuibile al fatto che Massimo e Gianluca, in un primo momento, non riescono ad accordarsi sul numero di sedie da destinare ai singoli spettacoli. ${ }^{22}$ Mentre per Massimo, per allestire lo spazio culturale Tedamis, non è indispensabile usare le sedie, Gianluca difende la tesi opposta: tutti gli spettacoli, compresi quelli che si svolgeranno nello spazio Tedamis, debbono essere muniti di sedie. Una volta deciso che anche lo spazio Tedamis dovrà disporre delle sedie, $i$ due non concordano sul genere di allestimento riservato a ciascuno spazio. Massimo, per semplificare il lavoro, vorrebbe proporre un unico allestimento da usare sia in piazza che al Tedamis ma Gianluca si oppone. Sarà Luca a decidere gli allestimenti dei due spazi.

Dal punto di vista metodologico, dopo aver identificato la sequenza, abbiamo proceduto alla trascrizione ${ }^{23}$ e quindi all'analisi a partire dai fenomeni conversazionali messi in evidenza dall'AC (SACKS; SCHEGLOFF; JEFFERSON, 1974), ovvero: discorsi simultanei, auto e etero interruzioni, pause, allungamenti vocalici, ripetizioni, cambi di topico, auto ed etero correzioni e segnali discorsivi. Inoltre, per identificare le funzioni svolte dai segnali discorsivi (d'ora in poi SD) ci siamo serviti della tassonomia, di matrice conversazionale, messa a punto da Bazzanella $(1994,1995,2011)$, che rappresenta il più importante studio condotto sui SD per l'italiano.

Tenendo conto che la conversazione è frutto di una collaborazione nel corso della quale i contributi del parlante di turno e dell'interlocutore si intrecciano, fino quasi a confondersi, la studiosa raggruppa i SD in due macrocategorie:

- I SD interazionali servono per sottolineare la dimensione interattiva della comunicazione. Sono prodotti dal parlante, cioè, colui che proferisce un determinato enunciato in quel dato momento e dall'interlocutore, cioè da colui che partecipa allo

\footnotetext{
${ }^{22}$ Gli spazi in cui si svolge il festival sono in tutto due: l'ampia piazza Torre di Berta, localizzata in pieno centro storico che ospita gli spettacoli più importanti; il centro culturale Tedamis, poco distante dal centro, che possiede un piccolo teatro destinato agli spettacoli collaterali.

${ }^{23}$ Le norme adottate sono quelle di Saks, Schegloff e Jefferson (1974).
} 
scambio comunicativo senza essere in possesso del turno in quel dato momento (e pertanto partecipa in silenzio o per mezzo di segnali non verbali o di SD specifici). Questi comprendono: i SD usati dal parlante per prendere il turno, richiamare l'attenzione, richiedere l'accordo o la conferma dell'interlocutore, i fatismi ${ }^{24}$ e i meccanismi di modulazione; ${ }^{25}$ i SD usati dalla parte dell'interlocutore per interrompere, confermare l'attenzione, dimostrare accordo, segnalare la ricezione.

- I SD metatestuali servono per organizzare il testo dal punto di vista dell'articolazione e della struttura argomentativa e comprendono i demarcativi, i focalizzatori e gli indicatori di riformulazione.

Si vedano le tabelle 1 e 2 che descrivono, per ciascuna macrocategoria, le funzioni dei $\mathrm{SD}$, seguite da alcune esemplificazioni.

TABELLA 1 - Funzioni interazionali dei segnali discorsiovi

\begin{tabular}{|l|l|}
\hline \multicolumn{1}{|c|}{ Dalla parte del parlante } & \multicolumn{1}{c|}{ Dalla parte dell'interlocutore } \\
\hline $\begin{array}{l}\text { Segnali relativi al turno: es. allora, ecco, } \\
\text { ma, } e\end{array}$ & $\begin{array}{l}\text { Meccanismi di interruzione: es. allora, } \\
\text { scusa, ma }\end{array}$ \\
\hline $\begin{array}{l}\text { Richiesta di attenzione: es. senti, dimmi, } \\
\text { mi segui? }\end{array}$ & Conferma di attenzione: es. davvero, $m h$ \\
\hline $\begin{array}{l}\text { Segnali relativi all'accordo: es. no? ti } \\
\text { pare? dico male? }\end{array}$ & $\begin{array}{l}\text { Conferma dell'accordo: es. perfetto, } \\
\text { assolutamente, come no }\end{array}$ \\
\hline $\begin{array}{l}\text { Controllo della ricezione: es. eh? } \\
\text { capisci? capito? }\end{array}$ & Fatismi: es. lo credo, so bene, povero \\
\hline Fatismi: es. come sai, sai, capisci & Back-channels: es. esatto, davvero, mh \\
\hline $\begin{array}{l}\text { Meccanismi di modulazione: es. } \\
\text { naturalmente, magari, se non mi sbaglio }\end{array}$ & \\
\hline
\end{tabular}

${ }^{24}$ Servono a sottolineare l'aspetto di "coesione sociale" della comunicazione (BAZZANELLA, 1994, p. 155).

${ }^{25}$ I meccanismi di modulazione sono usati per rafforzare o mitigare il contenuto proposizionale di un enunciato (BAZZANELLA, 1995, p. 238). 
TABELLA 2 - Funzioni metatestuali dei segnali discorsivi

\begin{tabular}{|l|l|}
\hline $\begin{array}{l}\text { Demarcativi: } \\
\text { organizzano il testo dal punto di vista } \\
\text { dell'articolazione e della struttura argomentativa }\end{array}$ & Poi, così, però, allora, quindi, perché \\
\hline $\begin{array}{l}\text { Focalizzatori: } \\
\text { usati dal parlante per segnalare i punti centrali } \\
\text { del discorso e possono indirizzare e regolare } \\
\text { l'elaborazione dell'informazione a livello cognitivo }\end{array}$ & Però, invece \\
\hline $\begin{array}{l}\text { Indicatori di riformulazione: } \\
\text { consistono in parafrasi, correzioni e esemplificazioni }\end{array}$ & Ciò̀, nel senso, voglio dire... \\
\hline
\end{tabular}

Successivamente, abbiamo proceduto all'identificazione e al conteggio dei tratti conversazionali, infine i dati sono stati trasferiti in una tabella per stabilirne la frequenza (tabella 3 ).

TABELLA 3 - Frequenza dei tratti conversazionali

\begin{tabular}{|l|c|}
\hline \multicolumn{1}{|c|}{ FENOMENI CONVERSAZIONALI } & FREQUENZA \\
\hline Segnali discorsivi & 12 \\
\hline Prolungamenti vocalici & 10 \\
\hline Discorsi simultanei & 5 \\
\hline Pause & 5 \\
\hline Ripetizioni & 3 \\
\hline Autocorrezioni & 2 \\
\hline Autointerruzioni & 2 \\
\hline Eterointerruzioni & 1 \\
\hline Eterocorrezioni & 0 \\
\hline Cambi di topico & 2 \\
\hline
\end{tabular}

\section{Analisi della microscena}

Vediamo ora nel dettaglio come si sviluppa la conversazione. Dal punto di vista organizzativo, la prima parte dell'interazione (dal 
turno 1 al turno 11), è costruita da una serie di scambi di opinioni, che seppur fra loro contrarie, si susseguono in maniera ordinata rispettando il cosiddetto Punto di Rilevanza Transizionale (SACKS; SCHEGLOFF; JEFFERSON, 1974). ${ }^{26}$

Esempio:

1 Massimo: credo invece che col tedamis con quelle che ((gesticolando)) cioè anche un allestimentino:: un pochino:: molto più:: speedy

2 Gianluca: no massimo aspetta un attimo le sedie al tedamis ci vanno messe ((congiungendo le mani)) la spiaggia ((guardando Luca)) è un'altra cosa eh? ((guardando Luca)) la spiaggia sono gli ombrelloni e::: ( giusto? ((guardando Luca))

3 Luca: ((mentre è occupato al computer)) sì

4 Gianluca: al tedamis le sedie quindi ci vanno messe

5 Massimo: sì però io le metterei qui le duecento sedie:: ((sollevando il braccio)) queste qui nostre (.) al massimo quelle veramente portate giù in extremis cioè in extremis chiaramente verificato ma non tutte quelle cinquecento che ((muovendo la testa)) che poi veramente non se sa dove mettere

6 Gianluca: ((avvicina la sedia al tavolo provocando un rumore)) le sedie più importanti sono quelle incatenate che sono esclusivamente per i tiromancino

7 Massimo: IO penserei così

8 Gianluca: non sono per odifreddi per odifreddi ci sono le sedie ci sono per tedamis e ci sono per i tiromancino

9 Massimo: Io odifreddi per me odifreddi scatta il festival ((allineando le mani)) quindi c'è l'allestimento festival (.) tedamis volevo dare un:: ((muovendo le mani)) un discorso diverso (.) volevo dare un taglio diverso ((gesticolando)) manco tanto ( ) nel senso

10 Gianluca: ((sorride))

11 Massimo: no però:: decidiamo:: insieme le forze le sappiamo tutti quanti quali sono quindi

Massimo nel turno 1 introduce il suo punto di vista attraverso l'uso del verbo credo, impiegato - come meccanismo di modulazione - seguito dal focalizzatore invece, usato per segnalare il punto centrale del discorso. In questo modo contribuisce ad aumentare il suo grado di impegno rispetto a quanto asserito. Segue un enunciato in cui omette l'oggetto della contesa, ovvero le sedie, e tenta di articolare la sua

\footnotetext{
${ }^{26}$ Punto in cui il turno può considerarsi potenzialmente completo e potrebbe verificarsi il passaggio di turno da un parlante all'altro.
} 
proposta attraverso: una riformulazione, introdotta dal SD ciò̀; dei prolungamenti vocalici; due meccanismi di modulazione un pochino e molto più. Il primo serve per mitigare la forza della sua proposta e il secondo per rafforzarla. Il turno 2 si apre con la negazione di Gianluca in risposta alla proposta di Massimo, accentuata dal meccanismo di modulazione - aspetta un attimo - che contribuisce a corroborare la sua contrarietà. Poi, con lo sguardo, e con i SD eh? e giusto?, cerca anche il consenso di Luca. Luca conferma con il SD sì il suo accordo, mentre continua a lavorare al computer. Allora Gianluca incalza e, ribadendo che è imprescindibile usare le sedie al Tedamis, impiega il SD quindi per concludere che devono essere usate (turno 4). L'inizio del turno 5 è una risposta di disapprovazione all' affermazione di Gianluca ed è costruita da una catena di SD (BAZZANELLA, 1995, p. 231). Con si Massimo accetta quanto asserito da Gianluca, con però aggiunge dell'altro, in questo modo si oppone all'affermazione del collega del turno 4, anche se poi mitigata dal condizionale metterei. Quest'ultimo è usato assieme ad altri meccanismi di modulazione, al massimo, veramente e chiaramente, per rafforzare la sua proposta (BAZZANELLA, 1995). Nel turno 6 Gianluca adotta nei confronti di Massimo un tono assertivo, enfatizzato dal SD esclusivamente, che aumenta l'autorità e conferisce potere al parlante, e dichiara che le sedie incatenate sono ad uso esclusivo del concerto dei Tiromancino. Massimo continua a ribadire la sua opinione aumentando il tono della voce, attenuato, però, dal condizionale penserei, che funge da modalizzatore, e dal SD cosi, con cui conclude la sua argomentazione (turno 7). Gianluca riafferma che le sedie serviranno per lo spettacolo di Odifreddi, per il Tedamis e per il concerto dei Tiromancino (turno 8). Massimo rafforza la sua opinione con il soggetto io, che mitiga immediatamente tramite il modulatore per me. Secondo lui, il festival vero e proprio inizia con lo spettacolo di Odifreddi e conclude con il $\mathrm{SD}$ quindi che, solo a partire da quel momento, scatterà l'allestimento festival. Dopo una breve pausa, riprende il discorso - intercalato da riformulazioni, ripetizioni e esitazioni e dal movimento delle mani - e ribadisce che l'allestimento che ha previsto per il Tedamis è diverso. Poi passa a esemplificare, con l'indicatore di riformulazione nel senso e il meccanismo di mitigazione manco tanto, in che cosa consiste. A questo punto la frase risulta incomprensibile a causa dei rumori (turno 9). Gianluca accoglie la proposta di Massimo con un sorriso che è non preferenziale rispetto alle attese di Massimo (turno 10). Massimo, 
che fino ad ora aveva cercato di contenere la sua posizione, passa ad esprime il proprio disappunto in maniera più esplicita. Prima usa il SD no però per controbattere, poi, per rafforzare la sua contrarietà, ricorre al meccanismo di modulazione sappiamo tutti quanti che conferisce alla frase autorità. Termina il turno con il SD quindi per reiterare la sua conclusione (turno 11).

Dal turno 12 al 21 i toni della discussione si accendono.

12 Gianluca: sì [però ((sfogliano il quaderno))

13 Massimo: [oppure s-[

14 Luca: (ride)) [a me mi piace quando lui se innervosisce ((imitando Gianluca)) sì ok ((ride))

15 Gianluca: non capisco non riesco a capire ((scrivendo nel quaderno)) che cosa

16 Massimo: GIANLU ALLORA DIMMI TU ((muovendo le mani)) perché a me a me sembra semplice

17 Gianluca: [NO NO

[dimmi tu

18 Massimo: [DIMMI TU? DIMMI TU? COME LA PENSAVI TU

19 Gianluca: [no:: io non la penso in nessum modo [io non riesco a capire ((congiunge le mani e muove la testa))

20 Massimo:

[NO PERCHÈ TU L'HAI

PENSATA tu l'hai pensato di sicuro

21 Gianluca: massimo io t'ho detto (.) ci vogliono le sedie per il tedamis io non ho pensieri nessuni

Nei turni 12, 13 e 14 si registra l'inizio di tre discorsi simultanei: quello di Gianluca in cui manifesta il suo disaccordo con il SD si però (turno 12); quello di Massimo che cerca di conquistare il turno con il $\mathrm{SD}$ oppure ma deve rinunciarvi, in quanto interrotto da Luca; quello di Luca che ottiene il turno e interviene in veste di mediatore per smussare i toni della polemica. Luca sposta la conversazione sul piano ironico e imita Gianluca quando si arrabbia (turno 14). Gianluca però non sta al gioco e dichiara il proprio disagio (turno 15). Massimo, sentendosi chiamato in causa, alza il tono della voce e anticipando il fatismo Gianlu che mitiga la forza del suo enunciato, chiede al collega una possibile soluzione (turno 16). La risposta di Gianluca però non è preferenziale e, sovrapponendosi al turno di Massimo, usa i SD no no, pronunciati con un tono più alto (turno 17). In questo modo si rifiuta di collaborare. Massimo, riallacciandosi al turno 16, si sovrappone al parlato di Gianluca e riformula la stessa domanda. La ripetizione contribuisce a enfatizzare 
la richiesta però è mitigata dai modulatori - a me - e - sembrare - (turno 18). Tra i turni 18 e 19 c'è un nuovo discorso simultaneo. Infatti Gianluca si sovrappone a Massimo per contestare, attraverso il rafforzatore io e la negazione no, accompagnati dal movimento del corpo, la sua premeditazione. Massimo, sovrapponendosi al turno 19, continua ad accusare Gianluca - con il rafforzamento di sicuro - di aver in mente un piano (turno 20). Gianluca formula la risposta alla domanda iniziata da Massimo nel turno 16 e con il modulatore - io t'ho detto - riafferma che ci vogliono le sedie (turno 21).

Dal turno 22 al 31 i toni della discussione si attenuano anche se le incomprensioni continuano.

22 Massimo: OK QUANTE?

23 Gianluca: quelle che tu pensi siano giuste per il tedamis d'acco- ovviamente non [cinquecento $=$

24 Massimo: [cento

25 Gianluca: $=$ cento sedie sono le stesse di odifreddi? ((guardando Massimo))

26 Massimo:allora (.) odifreddi per me ((toccandosi)) però magari ho capito male io per me scatta l'allestimento festival ((muovendo le mani)) quindi sdraio alberi è:: scusa piante

27 Gianluca: le piante ci sono quelle del tedamis

28 Massimo: allora allora vedi che è già diverso

29 Gianluca: [no per quello ( )

30 Massimo: [ALLORA ODIFREDDI è come tedamis sennò ragazzi ci ci complichiamo la vita è inutile no? Fare due tre quattro allestimenti se tu per odifreddi hai già:: a per:: per tedamis ci saranno già le piante

31 Gianluca: massi però aspetta un [attimo

32 Luca:

[sì ${ }^{\circ} \mathbf{s i}^{\circ}$ l'idea- l'unica cosa la differenza è che con odifreddi aggiungiamo le sdraio che ce l'abbiamo.

Massimo in un primo momento cede alla richiesta di Gianluca e chiede quante sedie occorrono al Tedamis, attraverso il SD ok, usato per esprimere accordo (turno 22). Anche Gianluca, con fare collaborativo, completa la seconda parte della coppia adiacente, proponendo a Massimo di stabilire lui un numero sufficiente di sedie. La disponibilità è espressa dal meccanismo di mitigazione quelle che tu pensi e dal SD d'accordo. Poi però si autointerrompe e attraverso l'indicatore di rafforzamento ovviamente, gli ricorda di non superare un certo limite (turno 23). In questo modo Gianluca manifesta la propria superiorità rispetto al collega. Ciò nonostante l'attitudine cooperativa di Massimo continua e, 
sovrapponendosi al turno di Gianluca, suggerisce cento sedie (turno 24). Gianluca domanda sequenzialmente a Massimo se le cento sedie saranno poi usate anche per allestire la piazza (turno 25). Massimo apre il turno con il SD allora, e spiega che, secondo lui, l'allestimento della piazza è diverso, perché composto da alberi e sdraio (turno 26). Per affermare la sua opinione utilizza però una serie di strategie che gli permettono di attenuare la forza del suo enunciato e quindi di evitare un ulteriore conflitto con Gianluca. Infatti, prima di esporre il suo parere, anticipa, ripetendolo due volte, il modulatore per me e con la frase però magari ho capito male io diminuisce l'impatto che l'affermazione potrebbe causare agli occhi di Gianluca. Gianluca aggiunge che le piante saranno quelle del Tedamis (turno 27). Massimo, in base all'informazione ricevuta nel turno 27, giunge ad un'ulteriore conclusione, che evidentemente è in conflitto con quella di Gianluca, e lo fa reiterando il SD allora, che ha una funzione consecutiva (turno 28). Gianluca interviene con il SD no per manifestare il disaccordo rispetto a Massimo (turno 29). Inizia un nuovo discorso simultaneo in cui Massimo si sovrappone al turno di Gianluca e, con il SD allora, ribadisce la sua conclusione. I due allestimenti dovranno essere identici. Poi usa il meccanismo di modulazione - sennò ragazzi - per conferire alla frase senso di appartenenza al gruppo e rafforzare quello che sta dicendo. Cerca anche la conferma di Gianluca con il SD no?. Il turno termina con una riformulazione (turno 30). Gianluca, che forse non condivide il ragionamento di Massimo, manifesta disappunto con il SD però, rafforzato dal modulatore aspetta un attimo (turno 31). Tuttavia non porta a termine il suo intervento perché interrotto da Luca che, a questo punto, si riallaccia al turno 30 di Massimo per asserire, con il SD sì sì, il suo accordo con la conclusione avanzata da Massimo. I due allestimenti sono identici, l'unica differenza è che per lo spettacolo di Odifreddi andranno aggiunte le sdraio. Con il suo intervento Luca esercita una forma di dominanza semantica (LINNEL; LUCKMANN, 1991; ORLETTI, 2000) e pone fine alla discussione fra i due, "imponendo" il suo punto di vista, non condiviso, in un primo momento, da Gianluca (turno 34) ${ }^{27} \mathrm{Nel}$ turno 35, rivolto a Gianluca, Luca, con una mossa non preferenziale, reitera, con un tono più alto della voce e due negazioni, la sua decisione finale, confermando la propria autorità (turno 35).

${ }^{27}$ Non possiamo confermare il dato, visto che il turno 34 risulta incomprensibile. 
Da questo momento, la conversazione si fa più distesa, e sviluppandosi principalmente con la struttura binaria domanda-risposta che si sussegue linearmente, è composta da turni molto più brevi, rispetto allo scambio precedente. A conferma del maggior coinvolgimento e cooperatività fra gli interattanti, diminuiscono i SD metatestuali, sostituiti dai SD interazionali.

Anche la divergenza d'opinioni fra Luca e Gianluca, relativa al numero delle sdraio che la compagnia possiede, si risolverà in appena tre turni, con l'accordo di Luca, espresso con il SD si va be ${ }^{28}$ (dal turno 36 al 39). Una volta superato l'equivoco di fondo e fissato il numero delle sdraio, l'interazione volgerà al termine con il consenso di Luca alla proposta di portare le sedie il giorno 14 (turno 44).

33 Massimo: per odifreddi quante sedie abbiamo previsto? ((guardando Gianluca))

34 Gianluca: ( )

35 Luca: NO NO s'è detto noi mettiamo le nostre ottanta sdraio

36 Gianluca: ((guardando Luca)) sessanta

37 Luca: ottanta sono?

38 Gianluca: sessanta sempre state sessanta

39 Luca: ${ }^{\circ}$ sì va be ${ }^{\circ}$ ((Gianluca sorride)) e::::e poi le le altre sedie non si mettono cioè si tengono accatastate dietro e poi si aggiungono se arriva il popolo

40 Massimo: quante? ce ne porto? ((guardando Luca con aria interrogativa))

41 Luca: un duecento almeno

42 Massimo: duecento

43 Gianluca: quindi se c'abbiamo già duecento sedie tanto vale portarcele il quattrordici

44 Luca: sì sì perfetto sì

\section{Risultati}

In termini di competenza interazionale, questo episodio ci offre diversi spunti per discutere le risorse sfruttate dai partecipanti e il loro effetto sull'avanzamento di questa discussione. Per prima cosa è evidente che, in generale, si tratta di uno scambio in cui i parlanti adottano un'attitudine poco collaborativa fra loro. Prova ne è la mancanza assoluta di back channels (SCHEGLOFF, 1981; BAZZANELLA,

${ }^{28}$ Il SD si va be' ha una duplice funzione: serve per prendere il turno e per sottolineare la parzialità dell'accordo (BAZZANELLA, 1994, p. 170). 
1994). Questi SD, dal punto di vista interazionale, sono una cartina tornasole per misurare il grado di coinvolgimento dei parlanti ai fini del proseguimento del discorso, dato che sono prodotti senza intenzione di prendere il turno ma per sostenere il parlante di turno (BAZZANELLA, 1994, p. 189). Se la prima parte dell'interazione (dal turno 1 al turno 11) è costruita da una serie di scambi di opinioni, che seppur fra loro contrarie, si susseguono in maniera ordinata e rispettando il cosiddetto Punto di Rilevanza Transizionale (SACKS; SCHEGLOFF; JEFFERSON, 1974), dal turno 12 al 21 la discussione si fa più intensa. La competitività si manifesta attraverso "discorsi simultanei" (BAZZANELLA, 1994, p. 177), sovrapposizioni e "interruzioni competitive" (BAZZANELLA, 1994, p. 189), realizzate dal parlante B, prima che il turno di A possa considerarsi completo. Questi elementi linguistici, associati alle "parole del corpo" (POGGI, 2006, p. 11) ${ }^{29}$ e a fattori prosodici, conferiscono alla conversazione una certa partecipazione emotiva e rivelano un evidente disaccordo fra gli interattanti. Tuttavia, il fenomeno che secondo noi è più rappresentativo di questo scambio è rappresentato da un'abbondante profusione di specifici SD che tessono la trama dell'interazione e puntellano gli interventi dei parlanti, al fine di affermare e controbattere le proprie opinioni. Nelle tabelle 4 e 5 abbiamo riportato, in base alla classificazione proposta da Bazzanella (1994, 1995, 2011), i SD interazionali e metatestuali usati da ciascun parlante, indicando turno, funzioni e frequenze. Le tabelle 6 e 7 indicano il totale delle occorrenze dei SD interazionali e metatestuali. Le liste che suggeriamo sono tutt'altro che chiuse ed esaurienti, visto che non è possibile attribuire una sola funzione ad uno specifico SD, ma piuttosto gradi diversi di funzionalità che agiscono a più livelli (BAZZANELLA, 2015, p. 38).

${ }^{29}$ Sono queste "le parole espresse dalle mani, dagli occhi, dal corpo, dal viso, dal movimento e dalla postura del busto e delle gambe, dal contatto fisico e dalle distanze che mettiamo fra noi e gli altri (POGGI, 2006, p. 11). 
TABELLA 4 - SD interazionali usati dai parlanti

\begin{tabular}{|c|c|c|c|}
\hline \multirow{8}{*}{$\begin{array}{l}\text { SD interazionali } \\
\text { usati da Massimo }\end{array}$} & Funzione & Tipo & Frequenza \\
\hline & $\begin{array}{l}\text { Meccanismo di } \\
\text { modulazione usato per } \\
\text { mitigare }\end{array}$ & $\begin{array}{l}\text { Un pochino (turno 1) } \\
\text { Al massimo (turno 5) } \\
\text { Io penserei (turno 7) } \\
\text { Manco tanto (turno 9) } \\
\text { Però magari (turno 26) } \\
\text { Per me (turno 9 e 26) } \\
\text { Io le metterei (turno 5) } \\
\text { Credo (turno 1) } \\
\text { Sembra (turno 16) }\end{array}$ & 9 \\
\hline & $\begin{array}{l}\text { Meccanismo di } \\
\text { modulazione usati per } \\
\text { rafforzare }\end{array}$ & $\begin{array}{l}\text { Molto più (turno 1) } \\
\text { Veramente (turno 5) } \\
\text { Chiaramente (turno 5) } \\
\text { Sappiamo (turno 11) } \\
\text { A me (turno 16) } \\
\text { Io (turno 9) } \\
\text { Di sicuro (turno 20) } \\
\text { Sennò ragazzi (turno 30) }\end{array}$ & 8 \\
\hline & $\begin{array}{l}\text { Accordo nei confronti } \\
\text { dell'interlocutore }\end{array}$ & Ok (turno 22) & 1 \\
\hline & Prendere il turno & $\begin{array}{l}\text { Oppure (turno 13) } \\
\text { Allora (turno 26) }\end{array}$ & 2 \\
\hline & Cedere il turno & Dimmi tu? (turno 18) & 1 \\
\hline & $\begin{array}{l}\text { Richiesta di conferma da } \\
\text { parte dell'interlocutore }\end{array}$ & No? (turno 30) & 1 \\
\hline & Fatismo & Gianlu (turno 16) & 1 \\
\hline \multirow[t]{4}{*}{$\begin{array}{l}\text { SD interazionali } \\
\text { usati da Gianluca }\end{array}$} & $\begin{array}{l}\text { Meccanismo di } \\
\text { modulazione usati per } \\
\text { rafforzare }\end{array}$ & $\begin{array}{l}\text { Esclusivamente (turno 6) } \\
\text { Ovviamente (turno 23) } \\
\text { Aspetta un attimo (turno } 2 \text { e } 31 \text { ) } \\
\text { Io (turno } 19 \text { e } 21 \text { ) } \\
\text { Io t'ho detto (turno 21) } \\
\text { Tu pensi (turno 23) }\end{array}$ & 8 \\
\hline & $\begin{array}{l}\text { Disaccordo nei confronti } \\
\text { dell'interlocutore }\end{array}$ & No (turno $2,17,19,29$ ) & 4 \\
\hline & Fatismo & Massi (turno 31) & 1 \\
\hline & $\begin{array}{l}\text { Richiesta di conferma da } \\
\text { parte dell'interlocutore }\end{array}$ & $\begin{array}{l}\text { Eh? (turno 2) } \\
\text { Giusto? (turno 2) } \\
\text { D'accordo (turno 23) }\end{array}$ & 3 \\
\hline \multirow[t]{2}{*}{$\begin{array}{l}\text { SD interazionali } \\
\text { usati da Luca }\end{array}$} & $\begin{array}{l}\text { Accordo nei confronti } \\
\text { dell'interlocutore }\end{array}$ & $\begin{array}{l}\text { Sì (turno } 3 \text { e 32) } \\
\text { Sì va be' (turno } 39 \text { ) } \\
\text { Sì sì perfetto sì (turno 44) }\end{array}$ & 3 \\
\hline & $\begin{array}{l}\text { Disaccordo nei confronti } \\
\text { dell'interlocutore }\end{array}$ & No no (turno 35) & 1 \\
\hline
\end{tabular}


TABELLA 5. SD metatestuali usati dai parlanti

\begin{tabular}{|c|c|c|c|}
\hline \multirow{8}{*}{$\begin{array}{l}\text { SD metatestuali } \\
\text { usati da Massimo }\end{array}$} & Funzione & Tipo & Frequenza \\
\hline & $\begin{array}{l}\text { Demarcativo con funzione } \\
\text { argomentativa }\end{array}$ & $\begin{array}{l}\text { Quindi (turno 9) } \\
\text { No però (turno 11) } \\
\text { No perché (turno 20) } \\
\text { Sì però (turno 5) }\end{array}$ & 4 \\
\hline & $\begin{array}{l}\text { Demarcativo con funzione } \\
\text { consecutiva }\end{array}$ & Allora (turno 16, 28) & 3 \\
\hline & $\begin{array}{l}\text { Demarcativo con funzione } \\
\text { conclusiva }\end{array}$ & $\begin{array}{l}\text { Così (turno 7) } \\
\text { Quindi (turno 11, 26) } \\
\text { Allora (turno 30) }\end{array}$ & 3 \\
\hline & $\begin{array}{l}\text { Demarcativo con funzione } \\
\text { aggiuntiva }\end{array}$ & Poi (turno 5) & 1 \\
\hline & $\begin{array}{l}\text { Demarcativo con funzione } \\
\text { finale }\end{array}$ & Perché (turno 16) & 1 \\
\hline & Indicatore di riformulazione & $\begin{array}{l}\text { Cioè (turno 1) } \\
\text { Nel senso (turno 9) }\end{array}$ & 2 \\
\hline & $\begin{array}{l}\text { Focalizzatore usato per } \\
\text { segnalare il punto centrale del } \\
\text { discorso }\end{array}$ & Invece (turno 1) & 1 \\
\hline \multirow{2}{*}{$\begin{array}{l}\text { SD metatestuali } \\
\text { usati da Gianluca }\end{array}$} & $\begin{array}{l}\text { Demarcativo con funzione } \\
\text { conclusiva }\end{array}$ & Quindi (turno 4, 43) & 2 \\
\hline & $\begin{array}{l}\text { Demarcativo con funzione } \\
\text { argomentativa }\end{array}$ & $\begin{array}{l}\text { Sì però (turno 12) } \\
\text { Però (turno } 31 \text { ) }\end{array}$ & 2 \\
\hline \multirow{2}{*}{$\begin{array}{l}\text { SD metatestuali } \\
\text { usati da Luca }\end{array}$} & $\begin{array}{l}\text { Demarcativo usato per } \\
\text { aggiungere }\end{array}$ & E poi (turno 39) & 2 \\
\hline & Indicatore di riformulazione & Cioè (turno 39) & 1 \\
\hline
\end{tabular}


TABELLA 6 - Scala di frequenza d'uso dei SD interazionali

\begin{tabular}{|c|l|l|l|l|l|c|}
\hline Modulatori & $\begin{array}{l}\text { Segnali di } \\
\text { disaccordo nei } \\
\text { confronti dell' } \\
\text { interlocutore }\end{array}$ & $\begin{array}{l}\text { Segnali di } \\
\text { accordo nei } \\
\text { confronti dell' } \\
\text { interlocutore }\end{array}$ & $\begin{array}{l}\text { Richiesta di } \\
\text { conferma da } \\
\text { parte dell' } \\
\text { interlocutore }\end{array}$ & Fatismi & $\begin{array}{l}\text { Presa di } \\
\text { turno }\end{array}$ & $\begin{array}{l}\text { Cedere } \\
\text { il turno }\end{array}$ \\
\hline 23 & 5 & 4 & 4 & 2 & 2 & 1 \\
\hline
\end{tabular}

TABELLA 7 - Scala di frequenza d'uso dei SD metatestuali

\begin{tabular}{|c|c|c|}
\hline Demarcativi & Indicatori di riformulazione & Focalizzatori \\
\hline 18 & 3 & 1 \\
\hline
\end{tabular}

Dall'analisi delle tabelle risulta che l'attitudine di Gianluca è senz'altro meno collaborativa rispetto a quella di Massimo, suo principale interlocutore. Questo è evidente dal numero di SD interazionali di disaccordo, prodotti da Gianluca e diretti a Massimo (4 frequenze). Un altro dato che richiama la nostra attenzione, e che ribadisce un forte grado di conflittualità, è la presenza di un unico SD di accordo, usato da Massimo. La mancanza di appoggio di Gianluca favorisce, a sua volta, l'insorgere di turni, tutto sommato molto più estesi e densi se paragonati a quelli di Gianluca, in cui Massimo si impegna a difendere le sue opinioni, attraverso una serie di strategie discorsive. Il discorso di Massimo è permeato da una fitta trama di SD demarcativi (poi, cosi, però, allora, quindi, perché), molto più ricca se paragonata a quella di Gianluca ${ }^{30}$ che, associati ad una vasta gamma di meccanismi di modulazione (credo, io penserei, io metterei, per me, magari, al massimo,veramente, chiaramente, di sicuro), ${ }^{31}$ contribuiscono - a seconda dell'intensità della voce - ad attenuare o a rinforzare i toni della discussione. Di tutt'altro tenore è la postura adottata da Gianluca che risulta essere molto meno cortese. Prova ne sia, oltre al già menzionato uso ricorrente del SD interattivo no, adoperato per manifestare il proprio disaccordo verso Gianluca, il ricorso a meccanismi di modulazione (come, ad esempio, esclusivamente, ovviamente, aspetta un attimo), che rafforzano il conteuto

\footnotetext{
${ }^{30}$ Il parlato di Massimo registra 16 occorrenze contro le 4 di Gianluca (tabella 5).

${ }^{31}$ In totale Massimo usa 17 modulatori (tabella 4).
} 
proposizionale dei suoi enunciati, conferendogli una certa autorità e potere. ${ }^{32}$ Quanto al ruolo svolto da Luca, seppur in termini di spazio sia marginale, è fondamentale; infatti, prendendo le difese di Massimo, attraverso il SD di accordo sì, e esprimendo il proprio disaccordo nei confronti di Gianluca con il SD no, sarà a lui decidere la disposizione finale delle sedie e a ristabilire un clima collaborativo fra Gianluca e Massimo.

\section{Conclusioni}

Questo articolo indaga in che modo è possibile reperire dati autentici, provenienti da conversazioni reali, che possano poi essere d'ausilio per costituire delle proposte didattiche specifiche per sviluppare, fra studenti d'italiano LS di livello avanzato, consapevolezza sulle convenzioni discorsive che regolano il comportamento quotidiano.

Il progetto, ancora in fieri, esplora i vantaggi che gli studi conversazionali possono offrire per osservare le strategie discorsive usate dai parlanti nel corso di una discussione, tratta da un dominio professionale. Alla prova dei fatti possiamo affermare che questo apparato metodologico si è rivelato particolarmente utile, in quanto, ha permesso di identificare i meccanismi discorsivi, usati in italiano, per affermare e controbattere le proprie opinioni. L'analisi ha evidenziato che i SD metatestuali e i modulatori svolgono un ruolo determinante perché, oltre a consentire di organizzare il testo dal punto di vista dell'articolazione e della struttura argomentativa, contribuiscono, assieme a elementi prosodici e prossemici, ad aumentare o diminuire il grado di conflittualità nel corso della discussione.

L'utilizzo del quadro applicativo nato in seno all'AC, attraverso percorsi di osservazione costituiti ad hoc, può quindi risultare una valida risorsa per esplorare l'input tratto dalla vita reale e sensibilizzare gli apprendenti allo sviluppo della competenza discorsiva in italiano LS, poiché, come abbiamo potuto vedere, consente di indagare nel dettaglio in che modo viene gestita l'interazione a livello locale dai parlanti.

Da un punto di vista applicativo, in questa sede, ci limitiamo a puntualizzare che è preferibile usare questo genere di input nei livelli

\footnotetext{
${ }^{32}$ Mentre i modulatori usati da Massimo sono più bilanciati: 9 servono per mitigare e 8 per rafforzare (tabella 4 ).
} 
avanzati, infatti; come mostrano gli studi aquisizionali, è solo nelle varietà interlinguistiche più avanzate che aumenta in modo significativo la gamma dei SD metatestuali con funzioni più ampie rispetto ai livelli basici (FERRARIS, 2004; JANFRANCESCO, 2015; MASCHERPA, 2016).

Una volta quindi riconosciute le potenzialità che l'input tratto dalla vita reale, riserva ai fini dell'insegnamento/apprendimento della LS, specie se decodificato attraverso gli strumenti messi a disposizione dall' $\mathrm{AC}$, la prossima sfida consisterà, e ciò sarà fatto in una seconda fase del progetto, nell'elaborare dei percorsi guidati che permettano a studenti di livello avanzato, mediante delle attività "coinvolgenti" (PERNAS et al., 2011, p. 132), di acquisire consapevolezza sulle norme discorsive in uso che regolano la lingua oggetto di studio. Questo, ci auguriamo, sarà realizzato a partire dall'approccio conversazionale, nato dall'incontro tra l'AC e la linguistica applicata e che si è rivelato particolarmente promettente sul piano didattico (cf., ad esempio, WONG, 2002; SEEDHOUSE, 2005; HUTH; TALEGHANI-NIKAZM, 2006; MORENO JAÉN; PÉREZ BASANTA, 2009).

\section{Bibliografia}

BAZZANELLA, C. Segnali discorsivi a confronto. Dati e teoria, un percorso integrato. In: BORREGUERO ZULOAGA, M.; GÓMEZJORDNA FERARY, S. (Org.). Marqueurs du discours dans le langues romanes: une approche contrastive. Limoges: Lambert-Lucas, 2015. p. 35-46.

BAZZANELLA, C. Segnali discorsivi. Treccani.it, Roma: Istituto Treccani La cultura italiana, 2011. Disponível em: $<$ http://www.treccani. it/> . Acesso em: 6/02/2017.

BAZZANELLA, C. I segnali discorsivi. In: RENZI, L.; SALVI, G.; CARDINALETTI, A. (Org.). Grande grammatica italiana di consultazione, v. III. Bolonha: Il Mulino, 1995. p. 226-257.

BAZZANELLA, C. I segnali discorsivi. In: BAZZANELLA, C. (Org.). Le facce del parlare. Firenze: La Nuova Italia, 1994. p.145-174.

BIRELlO, M.; VILAGRASA, A. Bravissimo!3 Corso d'italiano. Firenze: Bulgarini, 2014. 
BONAIUTO, M.; MARICCHIOLO, F. La comunicazione non verbale. Roma: Carocci, 2003.

CILIBERTI, A. La grammatica: modelli per l'insegnamento. Roma: Carocci, 2015.

CONTENTO, S. Attività bimodale: aspetti verbali e gestuali della comunicazione. In: GALOTOLO, R.; PALlOTTI, G. (Org.). La conversazione. Un'introduzione allo studio dell'interazione verbale. Milão: Raffaello Cortina Editore, 1999. p. 267-286.

DURANTI, A. Linguistic anthropology. Cambridge: Cambridge University Press, 1997. Doi: https://doi.org/10.1017/CBO9780511810190.

FERRARIS, S. Come usano $m a$ gli apprendenti di italiano L1 e L2?. In: $3^{\circ}$ CONGRESSO DI STUDI DELL'ASSOCIAZIONE ITALIANA DI LINGUISTICA APPLICATA. 2002, Perugia. Atti del $3^{\circ}$ congresso di studi dell 'Associazione Italiana di Linguistica Applicata. Perugia: Guerra Edizioni, 2004, p.73-91.

FERRONI, R.; BIRELLO, M. Meta-analisi e applicazione di una proposta didattica orientata all'azione per l'apprendimento dei segnali discorsivi in italiano LS. Italiano LinguaDue, Milão, v. 1, n. 8, p.30-53, 2016.

GALATOLO, R.; PALLOTTI, G. Introduzione. In: GALATOLO, R.; PALLOTTI, G. (Org.). La conversazione. Un'introduzione allo studio dell'interazione verbale. Milão: Raffaello Cortina Editore, 1999. p. 11-19.

GILLANI, E.; PERNAS, P. La trasmissione del parlato nei libri di testo di italiano LS: dall'oralità madrelingua all'input orale. In: XII CONGRESSO SILFI SOCIETÀ INTERNAZIONALE DI LINGUISTICA E FILOLOGIA ITALIANA, 2012, Helsinki. Atti del XII Congresso SILFI Società Internazionale di Linguistica e Filologia Italiana. Florença: Franco Cesati Editore , 2014. p. 461-469.

HUTH, T.; TALEGHANI-NIKAZM, C. How can insights from conversation analysis be directly applied to teaching L2 pragmatics?. Language Teaching Research, Oxford, v. 10, n.1, p. 53-79, 2006. Doi: https://doi.org/10.1191/13621688061r184oa.

JANFRANCESCO, E. L'acquisizione dei segnali discorsivi in italiano L2. Italiano LinguaDue, Milão, v. 7, n. 1, p. 1-39, 2015. 
LINELL, P.; LUKMANN, T. 1990, Asymmetries in dialogue: some conceptual preliminaries. In: MARKOVÁ, I.; FOPPA, K. (Org.). The dynamics of dialogue. Harvester Wheatsheaf: Hemel Hempstead, 1990. p. 1-20.

MASCHERPA, E. I segnali discorsivi allora, quindi, però, ma in apprendenti di italiano L2. Cuadernos de Filología Italiana, Madri, v. 23, n.1, p.119-140, 2016.

MORENO J. M.; PÉREZ BASANTA, C. Developing conversational competence through language-awareness and multimodality: the use of DVDs. ReCALL, Cambridge, v. 21, n.3, p. 283-301, 2009. Doi: https:// doi.org/10.1017/S0958344009990036.

ORLETTI, F. La conversazione diseguale: potere e interazione. Roma: Carocci, 2000.

PEKAREK D. S.; POCHON-BERGER, E. The development of L2 interactional competence: evidence from turn-taking organization, sequence organization, repair organization and preference organization. In: CADIERNO, T.; ESKILDSEN, S. (Org.). Usage-based perspectives on second language learning. Berlim: Mouton De Gruyter, 2015. p. 233-267.

PERNAS, P.; GILLANI, E.; CACCHIONE, A. Costruire testi, strutture conversazionali: la didattica dei segnali discorsivi come elementi pivot dell'interazione verbale. Italiano LinguaDue, Milano, v. 3, n. 1, p. 65138, 2011.

POGGI, I. Le parole del corpo. Introduzione alla comunicazione multimodale. Roma: Carocci, 2006.

PUGLIESE, R. Figurati, tra i segnali discorsivi. Una prospettiva pedagogica In: PISTOLESI, E.; PUGLIESE, R.; FIVELLA, B. (Org.). Parole, gesti, interpretazioni. Studi linguistici per Carla Bazzanella. Roma: Aracne, 2015, p.163-207.

QUADRO COMUNE EUROPEO DI RIFERIMENTO PER LE LINGUE (QCER): apprendimento insegnamento valutazione. Firenze: La Nuova Italia-Oxford, 2002. 
SACKS, H.; SCHEGLOFF, E.; JEFFERSON, G. A simplest systematic for the organization of turn-taking in conversation. Language, v. 50, n. 4, p. 696-735, 1974. Doi: https://doi.org/10.1353/lan.1974.0010.

SCHEGLOFF, E. Discourse as an interactional achievement: some uses of 'uh huh' and other things that come between sentences. In: TANNEN, D. (Org.). Analzing discourse: text and talk. Washington: D. C, 1981, p. 71-93.

SEEDHOUSE, P. Conversation Analysis and language learning. Language Theaching, Oxford, v. 38, n. 4, p. 223-262, 2005. Doi: https:// doi.org/10.1017/S0261444805003010.

ZORZI, D. Contributi dell'analisi della conversazione all'insegnamento dell'italiano L2. In: MAGGINI, M. (Org.). Atti del III congresso ILSA, Comune di Firenze, 1996, p. 11-39.

WONG, J. 'Applying' conversation analysis in applied linguistics: evaluating dialogue in English as a second language textbooks. International Review of Applied Linguistics, v. 40, n. 1, p. 37-60, 2002. Doi: https://doi.org/10.1515/iral.2002.003.

YOUNG, R. Interactional competence in language learning, teaching, and testing. In: HINKEL, E. (Org.). Handbook of research in second language teaching and learning. Londres; Nova York: Routledge, 2011, p. 426-443. 